\title{
Indicadores e a análise da oferta cultural na Cidade do Rio de Janeiro
}

Daniele C. Dantas ${ }^{1}$

\section{RESUMO}

0 uso de dados e indicadores na gestão e nas políticas culturais é uma prática em ascensão no século XXI. Recurso estratégico e apoio para processos de tomada de decisão, o uso de indicadores ajuda a confirmar aspectos e percepções sobre dinâmicas e práticas, bem como evidenciar outros não percebidos. Tomando como referência as contribuições do uso de referenciais para mensuração de aspectos da gestão cultural, o presente artigo tem como objeto o Indicador de Oferta Cultural (IOC) na cidade do Rio de Janeiro a partir de dados de equipamentos sob a gestão da Secretaria Municipal de Cultura do Rio de Janeiro (SMC-RJ), para o ano de 2013, proposto na pesquisa realizada no mestrado em Estudos Populacionais e Pesquisas Sociais, na Escola Nacional de Ciências Estatísticas (ENCE/IBCE), em 2015. Tem como objetivos apresentar a proposta metodológica e os resultados do indicador proposto, em duas escalas administrativo-geográficas (Área de Planejamento e Região Administrativa); além de considerações sobre um sistema complementar de indicadores e as contribuições do uso de dados e indicadores nos processos de tomada de decisão na gestão cultural. São utilizados dados de registro administrativo da SMC-RJ sobre atividade, capacidade e público dos cinqüenta e dois equipamentos culturais sob a gestão da Secretaria, entre os meses de janeiro e dezembro de 2013. Foi utilizado o Excel, do pacote Office da Microsoft, para a construção dos indicadores, a partir de técnicas estatísticas; com apoio do ArcGIS v.10 para aplicação de técnicas de geoprocessamento para a espacialização dos resultados. Os resultados confirmam diferenças na distribuição da oferta cultural em diferentes regiões da cidade, afirmando contribuições do uso de dados e indicadores na avaliação de aspectos gerenciais e possibilidades de apoio a processos de tomada de decisão, reconhecido o uso estratégico de dados do cotidiano na gestão.

PALAVRA-CHAVE: Dados; Indicadores culturais; Oferta cultural; Política cultural Rio de Janeiro.

\section{ABSTRACT}

The use of data and indicators in management and cultural policies is a rising practice in the 21st century. Strategic use and support for decision-making processes, the use of indicators helps to confirm aspects and perceptions about dynamics and practices, as well as evidence of others not perceived. Taking as reference the contributions of the use of reference frameworks to measure aspects of cultural management, this article has as object the Cultural offer Indicator (IOC) in the city of Rio de Janeiro based on equipment data under the management of the Municipal Secretary of Rio de Janeiro (SMC$\mathrm{RJ})$, for the year 2013, proposed in the research developed out in the master's degree in Population Studies and Social Research, at the National School of Statistical Sciences (ENCE / IBCE), in 2015. Its objectives are to present the methodological proposal and the results of the proposed indicator, in two administrative-geographical scales (Planning Area and Administrative Region); as well as considerations on a complementary system of indicators and the contributions of the use of data and indicators in decision-making processes in cultural management. Administrative data of the SMC-RJ on the activity, capacity and public of the fifty-two cultural equipment under the Secretariat's management, between January and December 2013, were used. Excel was used, from the Microsoft Office suite, for the construction of the indicators, based on statistical techniques; with support from ArcGIS v.10 for the application of geoprocessing techniques for spatialization of results. The results confirm differences in the distribution of cultural offer in different regions of the city, affirming contributions of the use of data and indicators in the evaluation of management aspects and possibilities of support to decision making processes, recognizing the strategic use of daily data in management.

KEY-WORDS: Data; Cultural indicators; Cultural offer; Cultural policy; Rio de Janeiro

1 Doutoranda em Ciência da Informação (UFRJ/IBICT), Mestre em Estudos Populacionais e Pesquisas Sociais (ENCE/IBGE) danielecdantas@gmail.com 


\section{Introdução}

A orientação da gestão por dados é realidade em diferentes áreas e perfis institucionais, apresentando-se como recurso estratégico e diferencial competitivo destacado tanto nos ambientes de negócio privados quanto na gestão pública. Há pouco mais de duas décadas, a gestão cultural brasileira vem se apropriando mais do uso de dados, buscando reuni-los, organizá-los e sistematizá-los para a melhor comunicação de aspectos e resultados de suas ações, através de indicadores.

Identifica-se o uso de referenciais quantitativos no processamento e apresentação de resultados como meio de contribuir para a leitura e percepção de aspectos e cenários também na gestão cultural, oferecendo dispositivos mais acurados para tomadas de decisão de curto, médio e longo prazos aos gestores. Estas possibilidades podem ser verificadas com a construção e aplicação de indicadores sobre diferentes aspectos, como a análise da oferta cultural em um recorte definido a partir das condições dos dados disponíveis.

Em 2015, propôs-se a construção de um indicador sintético para analisar a oferta cultural nas diferentes regiões da cidade do Rio de Janeiro (por Áreas de Planejamento e Região Administrativa). Naquele momento, a disponibilidade de dados de registros administrativos referentes ao ano de 2013, consolidados por um departamento da gestão municipal de cultura, foi oportuna em um ambiente favorável, particularmente por, neste ambiente, estes dados estarem organizados e se ter acesso a eles.

Assim, desenvolveu-se um indicador sintético sobre a distribuição da oferta cultural em equipamentos sob a gestão da Secretaria Municipal de Cultura do Rio de Janeiro (SMC-RJ) e, considerando a pertinência, apresentou-se um conjunto de indicadores complementares para apoio à compreensão de um cenário mais complexo e detalhado da oferta cultural em análise. A ampliação do escopo visava a apresentação de um conjunto de indicadores que não se limitasse a evidenciar em que regiões ou equipamentos culturais públicos da cidade a oferta fora maior naquele ano de 2013, mas também identificar meios para que se compreendessem aspectos que permitissem conhecer como estava composto o cenário da oferta cultural no território.

A construção dos indicadores complementares oferece mais possibilidades de leitura de cenário. Possibilidades de usar a informação para compreender como a capacidade e as atividades realizadas contribuíram para a resposta final que confirma o desequilíbrio na distribuição da oferta cultural nas diferentes regiões (nas diferentes Áreas de Planejamento e Regiões Administrativas), além de permitir fazer análises similares sobre os equipamentos culturais individualmente. Possibilidades de uso da informação também ajudando a reconhecer que mesmo em Áreas de Planejamento (APs) onde se verificavam maior concentração de oferta cultural existiam Regiões Administrativas (RAs) com pouca ou nenhuma disponibilidade de oferta.

Assim, o presente artigo tem como objeto a análise da distribuição da oferta cultural na cidade do Rio de Janeiro em equipamentos culturais sob a gestão da Secretaria Municipal de Cultura (SMC-RJ) em 2013 e tem como objetivos apresentar os indicadores propostos e pontuar reflexões sobre o uso de dados públicos na produção de informações que possam subsidiar processos de tomadas de decisão pelos gestores. Para isso, foram utilizados dados cedidos pela SMC-RJ sobre o potencial de atender demandas e usos dos equipamentos culturais (a partir de dados sobre capacidade, atividades realizadas e público atendido) entre os meses de janeiro e dezembro de 2013. Os indicadores foram construídos a partir de técnicas estatísticas com uso do Excel, do pacote Office da Microsoft, com apoio de técnicas de geoprocessamento, com ArcGIS v.10, para a espacialização dos resultados.

Na primeira seção são apresentados conceitos centrais sobre indicadores e sua aplicação no contexto da gestão e das políticas públicas, além da fundamentação teórica que dá 
base à proposta metodológica e de reflexões sobre o uso de indicadores. Contexto sóciodemográfico e histórico sobre a cidade do Rio de Janeiro e a infraestrutura para oferta cultural sob a gestão da Secretaria Municipal de Cultura são apresentados na segunda seção. Na terceira seção, apresentam-se informações sobre a fonte e o conjunto de dados utilizados, bem como a proposta metodológica do Indicador de Oferta Cultural (IOC) e considerações sobre os indicadores do sistema complementar. Segue, na quarta seção, a apresentação dos resultados do IOC por Área de Planejamento e Região Administrativa, considerações sobre os resultados por ambos os recortes administrativo-geográficos com ponderações sobre o uso oportuno de indicadores buscando a otimização dos resultados e recursos, assim como o alcance das atividades culturais pelos diferentes públicos da gestão cultural. Por fim, resultados confirmam diferenças na distribuição da oferta cultural em diferentes regiões da cidade e as considerações apontam contribuições do uso de dados e indicadores para a apreciação de aspectos gerenciais e busca por referenciais que apóiem tomadas de decisão.

O resultado do trabalho favorece o reconhecimento da contribuição do uso de dados na produção de informações com vistas a instrumentalizar a ação dos gestores no território. Propõe-se que o processo de construção dos indicadores, a partir do reconhecimento do valor estratégico dos dados produzidos no cotidiano da gestão, apóie a identificação de oportunidades para a adesão e incremento de processos que fortaleçam as tomadas de decisão de gestores culturais, pautadas em dados e informações sobre a realidade na e para a qual estão designados atuar.

\section{Referenciais teóricos}

A base conceitual para a construção dos indicadores propostos e as análises sobre oferta cultural desenvolvidas no presente trabalho esta pautada no conceito ampliado de cultura, adotado pelo UNESCO (2002), considerando os traços subjetivos e objetivos, intelectuais, morais e emocionais de um grupo, contemplando as expressões artísticas, modos vida e convivência, sistemas de valores, crenças e tradições (UNESCO, 2002). O escopo do conceito permite aproximar a compreensão das práticas culturais no contexto da expressão de pessoas e grupos em um processo de práticas relacionadas aos meios de expressão artísticos e formas de expressão (COELHO, 2004). Estão contempladas aí as produções audiovisuais, musicais e teatrais, exposições de arte e manifestações de culturas tradicionais e populares, entre outras.

Dessa maneira, por oferta cultural compreendem-se as atividades realizadas em equipamentos culturais sob a gestão da Secretaria Municipal de Cultura do Rio de Janeiro ou atividades diretamente vinculadas a estes espaços. Compreendem-se equipamentos culturais como sinônimos de espaço cultural, centro cultural ou casa de cultura, ou seja, edificações destinadas a práticas culturais (teatros, cinemas, bibliotecas, centros de cultura, filmotecas, museus).

[...] Lugar em que se oferece a possibilidade de produzir-se ou consumir-se diferentes modalidades culturais (COELHO, 2004, p. 165-166).

Considerando a gestão de um conjunto de equipamentos culturais, os processos de tomada de decisão dos gestores pautados no uso de dados e construção de indicadores são práticas que podem ser adotadas em diferentes níveis de gestão. Tendo em vista que o indicador cultural pode ser considerado um tipo de indicador social, ele representará um fenômeno da realidade social dotada de sentido e comportando sua leitura em determinado contexto, pautada em um conceito que traduza o fenômeno em análise, limitado pelo escopo conceitual e pelos dados disponíveis (JANNUZZI, 2004). Assim, um indicador cultural pode ser compreendido como um recurso para mensuração de aspectos 
do campo cultural, através do qual seja possível representar medidas construídas sobre o contexto em análise. Compete ao usuário deste recurso reconhecer possíveis limitações (maiores ou menores) na tradução de alguns aspectos, comumente mais subjetivos, para a forma quantitativa, quando for o caso (DANTAS, 2017).

Para o uso de indicadores culturais na gestão, como recursos de apoio à compreensão e análises sobre a realidade observada, três abordagens conceituais apresentam perspectivas complementares. De acordo com Yue et. al. (2011) indicadores culturais são estatísticas que dão suporte ao monitoramento para o planejamento cultural contextualizando os fenômenos culturais existentes; Pfenniger (2004) os considera instrumentos que fornecem informações relevantes para as políticas culturais; e Fanchette (1979) afirma que são ferramentas que auxiliam na definição de problemas e no desenho de tendências culturais orientando o planejamento cultural.

Considerando o bom uso dos indicadores como recurso de apoio à gestão, é importante ter atenção às possibilidades metodológicas no tocante à construção das escalas apropriadas e à escolha ou construção do conceito que o indicador traduzirá (DANTAS, 2017). É importante ainda ter atenção ao que Piovani (2015) chama de estiramento semântico do conceito de medição, que representa a tendência em forçar a quantificação de aspectos qualitativos que implicam em atribuições de rótulos numéricos a quaisquer métodos que gerem uma medida (PIOVANI, 2015). Tais cuidados representam aspectos centrais nos processos que envolvam propostas de medição de aspectos qualitativos, comuns no campo cultural. Analisando os resultados de forma atenta, os indicadores sinalizarão ao gestor que alguns aspectos da gestão podem necessitar de acompanhamento mais de perto, favorecendo a percepção de aspectos sensíveis, bem como de elementos para as tomadas de decisão pautadas em dados da realidade.

O cenário que favoreceu e mobilizou a adesão a práticas que levaram à incorporação do uso de indicadores na gestão cultural apresenta marcos com a publicação de estudos realizados em diferentes estados brasileiros, assinaturas de acordos e termos de cooperação entre o Ministério da Cultura e órgãos com expertise no desenvolvimento de estudos e usos de informações quantitativas, como o Instituto Brasileiro de Geografia e Estatísticas (IBGE). A estruturação do Sistema Nacional de Informações e Indicadores Culturais (SNIIC) também foi uma ação do Ministério da Cultura desenvolvida em um contexto de valorização do uso de informações sistematizadas no planejamento e na gestão pública de cultura com marcos legais, através dos quais se buscou criar um ambiente institucional favorável.

Este ambiente institucional beneficia o uso da informação adicionando valor aos argumentos na gestão cultural. Neste ambiente, dados e informação tornam-se ativos estratégicos, considerando-se melhores condições para sua obtenção, além do decrescimento dos custos ampliando o uso da informação nos processos de gestão e produção de valor (CAVALCANTI; GOMES, 2000; 2001). Práticas comuns com benefícios reconhecidos desde a metade do século XX em outras áreas, o uso de dados, informação e indicadores como instrumento de gestão de políticas públicas avançou, nos primeiros anos do século XXI, no contexto da gestão cultural.

Médici (1990) ratifica a importância do uso de dados e informação no planejamento e destacando a valorização do planejamento e a demanda por mais informações, ressaltando que a informação é fundamental para o planejamento (MÉDICl, 1990). Reconhece-se que a adoção de práticas que compreendem o uso de indicadores culturais possibilita o aprimoramento de processos de planejamento e gestão de políticas públicas.

Em contexto ampliado, Morais e Lima (2001) afirmam que indicadores são instrumentos importantes para auxiliar os formuladores de políticas a definir as prioridades sociais (MORAIS; LIMA, 2001, p. 25). Os autores advogam pela importância do uso dos indicadores na gestão, como ferramenta de suporte ao processo de tomada de decisão, visto que são 
particularmente úteis para orientar as ações nas diversas esferas do governo, configurandose em insumos indispensáveis para o planejamento de políticas e programas (MORAIS; LIMA, 2001, p 21). Os indicadores são reconhecidos como ferramentas relevantes para a gestão em função das contribuições para sua melhoria efetiva em um cenário de valorização do uso de dados e informação na gestão cultural.

Do tipo de informação necessária, identificam-se as fontes disponíveis através de indicadores já prontos ou de dados que possibilitem a construção de indicadores que tragam as contribuições necessárias. O paradoxo da necessidade de dados e da ausência ou dificuldade para a obtenção deles é salientada por Jannuzzi (2005) para todas as etapas do ciclo de políticas públicas, visto que

[...] uma das grandes dificuldades atuais no acompanhamento de programas públicos é dispor de informações periódicas e específicas acerca do processo de sua implementação e do alcance dos resultados e do impacto social que tais programas estão tendo nos segmentos sociodemográficos ou nas comunidades focalizadas por eles (JANNUZZI, 2005, p. 157).

Isso ratifica a necessidade de os gestores culturais buscarem obter os dados relevantes para sua gestão, seja pela contratação de serviços de coleta de dados, seja pela adoção de práticas gerenciais de organização dos dados de registros administrativos da própria gestão. Em um contexto de produção de dados incipiente na gestão cultural brasileira, o uso de dados de registros administrativos traduz-se em vantagens por tender a ter cobertura ampla e completa do objeto no período, boas chances de apresentar dados e metadados mais detalhados permitindo análises em diferentes escalas e, em geral, custo baixo (WALLGREN; WALLGREN, 2012). Desta forma, apresenta-se como um investimento necessário e vantajoso na busca por benefícios de médio e longo prazos.

Neste contexto, tanto o uso de dados, informações e indicadores, quanto o reconhecimento das contribuições em uma gestão que busca otimizar os processos de tomada de decisão são centrais em uma gestão pautada em conteúdos que representem a realidade onde está inserida e atua. Isso pode dar potência aos resultados previstos nos objetivos e favorecer melhorias contínuas na gestão e nas entregas ao público.

\section{Rio de Janeiro e a infraestrutura pública para fruição cultural na cidade em 2013}

Capital do Brasil Império até a mudança da capital da República para Brasília, nos anos 1960, o município do Rio de Janeiro (capital do estado de mesmo nome) registrava população de, aproximadamente, 6.320 .446 habitantes, composta por $53 \%$ de mulheres e $47 \%$ de homens ${ }^{2}$, de acordo com o Censo 2010, vivendo em uma área de 1.224,56 km². O Rio de Janeiro guarda historicamente parte importante da infraestrutura para oferta de serviços culturais do país, herdada do período de construção do país no qual ambientou etapas de seu desenvolvimento econômico, político, social e cultural.

Cidade com expressiva oferta cultural, o Rio de Janeiro sedia grupos e instituições artísticas de destaque, além de grupos de mídia e comunicação, como gravadoras de música, produtoras e distribuidoras de audiovisual e emissoras de televisão. O investimento em ações culturais na cidade também é expressivo. A presença de empresas de portes e tipos de atividade econômica variados, a freqüência turística nacional e internacional e a representação

2 Fonte: INSTITUTO BRASILEIRO DE GEOGRAFIA E ESTATÍSTICA. Disponível em <www.ibge.go v.br> acesso em 06 dez 2014.

3 Fonte: INSTITUTO MUNICIPAL DE URBANISMO PEREIRA PASSOS - IPP-RJ. Armazém de Dados. Disponível em <http://www.armazemdedados.rio.rj.gov.br> acesso em 06 dez 2014. 
icônica da imagem do Brasil no mundo são fatores que compõem um ambiente mobilizador e catalisador da intensa oferta e fruição cultural na cidade. Nesse ambiente, a infraestrutura para usos culturais na cidade é composta por equipamentos privados e públicos; sob gestão federal, estadual e municipal (distribuídos em diferentes secretarias e fundações); além de equipamentos culturais vinculados a organizações sociais e coletivos artísticos.

Em 2013, a divisão administrativa da cidade era composta por cinco Áreas de Planejamento com dezesseis Regiões de Planejamento e trinta e três Regiões Administrativas, onde se inscreviam cento e sessenta bairros ${ }^{4}$. De acordo com dados do Instituto Municipal de Urbanismo Pereira Passos (IPP-RJ), autarquia pública da cidade, em 2008, a cidade contava seiscentos e sessenta e um equipamentos culturais sob a gestão municipal (por fundações públicas e outras secretarias), estadual e federal, além de equipamentos privados, entre museus, bibliotecas, teatros, salas de cinema, galerias, espaços e centros culturais, escolas e sociedades musicais. Sob a gestão municipal, os equipamentos culturais estão distribuídos entre secretarias municipais e outros perfis de órgão da gestão pública na cultura, turismo, educação, ciência e tecnologia, por exemplo ${ }^{5}$.

Tendo em vista a observação de equipamentos culturais sob o mesmo perfil de gestão, os dados da SMC-RJ oferecem informações que permitem avaliar espaços sob uma orientação gerencial e política mais alinhada e uniforme. Tais espaços totalizavam cinquenta e duas unidades, naquele ano, sendo quatro Arenas Culturais, doze Bibliotecas, dez Centros Culturais, dez Lonas Culturais, quatro Museus e doze Teatros. A construção ou incorporação deles ao conjunto de equipamentos culturais da Secretaria Municipal de Cultura da cidade acompanhou políticas das diferentes gestões, especialmente nas últimas décadas, continuando nos anos seguintes a 2013.

Quanto à distribuição dos espaços para a oferta de serviços culturais na cidade, observase a concentração de equipamentos culturais em algumas regiões, nas diferentes Áreas de Planejamento (APs) e Regiões Administrativas (RAs). Eles estão presentes em todas as Áreas de Planejamento, mas não em todas as Regiões Administrativas, verificando-se também que nem todas as RAs e APs têm todos os tipos de equipamento cultural.

Este cenário suscita reflexões e mobiliza o interesse por compreender aspectos que evidenciem mais do que o desequilíbrio explicitado, inicialmente, pela distribuição da oferta de infraestrutura de serviços culturais, também verificada em outros recortes regionais e bairros da cidade, desde os anos 1990. Na Figura 1, é possível ver a distribuição espacial destes equipamentos culturais.

Para a melhor compreensão dos resultados do Indicador de Oferta Cultural, contribui conhecer as especificidades dos diferentes equipamentos culturais deste conjunto, bem como informações sobre sua localização nos diferentes bairros da cidade, como apresentado a seguir.

As Arenas Culturais são espaços utilizados nos formatos arena e palco italiano com possibilidade de integração com o entorno através de aberturas na plateia e no palco. São co-geridos por instituições selecionadas por licitação. Em 2013, integravam os equipamentos da SMC-RJ as Arenas Cariocas Jovelina Pérola Negra, na Pavuna; Dicró - Carlos Roberto de Oliveira, na Penha; Abelardo Barbosa - Chacrinha, na Pedra de Guaratiba; e Fernando Torres, em Madureira (SMC, 2013).

4 Fonte: RIO DE JANEIRO. Regiões Administrativas do Rio de Janeiro. Disponível em <http://www.rio.r j.gov.br/web/smg/regioes-administrativas> acesso em 06 dez 2014.

Hoje, a divisão administrativa da cidade do Rio de Janeiro totaliza cento e sessenta e dois bairros. Fonte: INSTITUTO MUNICIPAL DE URBANISMO PEREIRA PASSOS - IPP-RJ. Cidade. Disponível em <http:// portalgeo.rio.rj.gov.br/bairroscariocas/index_cidade.htm> acesso em 28 jan 2018. 
As Bibliotecas são equipamentos culturais com acervo que atende a faixas etárias distintas, disponíveis para consulta e empréstimo pela internet e realização de atividades culturais de incentivo à leitura. No ano de 2013, integravam os equipamentos da SMC-RJ Bibliotecas em Botafogo, Campo Grande, Gamboa (fechada para obras, mas funcionando temporariamente no Centro de Artes Calouste Gulbenkian), Ilha do Governador, Irajá, Jacarapaguá, Tijuca, Cidade Nova, Maré, Guandu (fechada para obras naquele ano), Santa Teresa e a biblioteca volante (sediada na Biblioteca Popular de Irajá) (SMC, 2009).

Figura 1: Infraestrutura cultural sob a gestão da Secretaria Municipal de Cultural do Rio de Janeiro, 2013

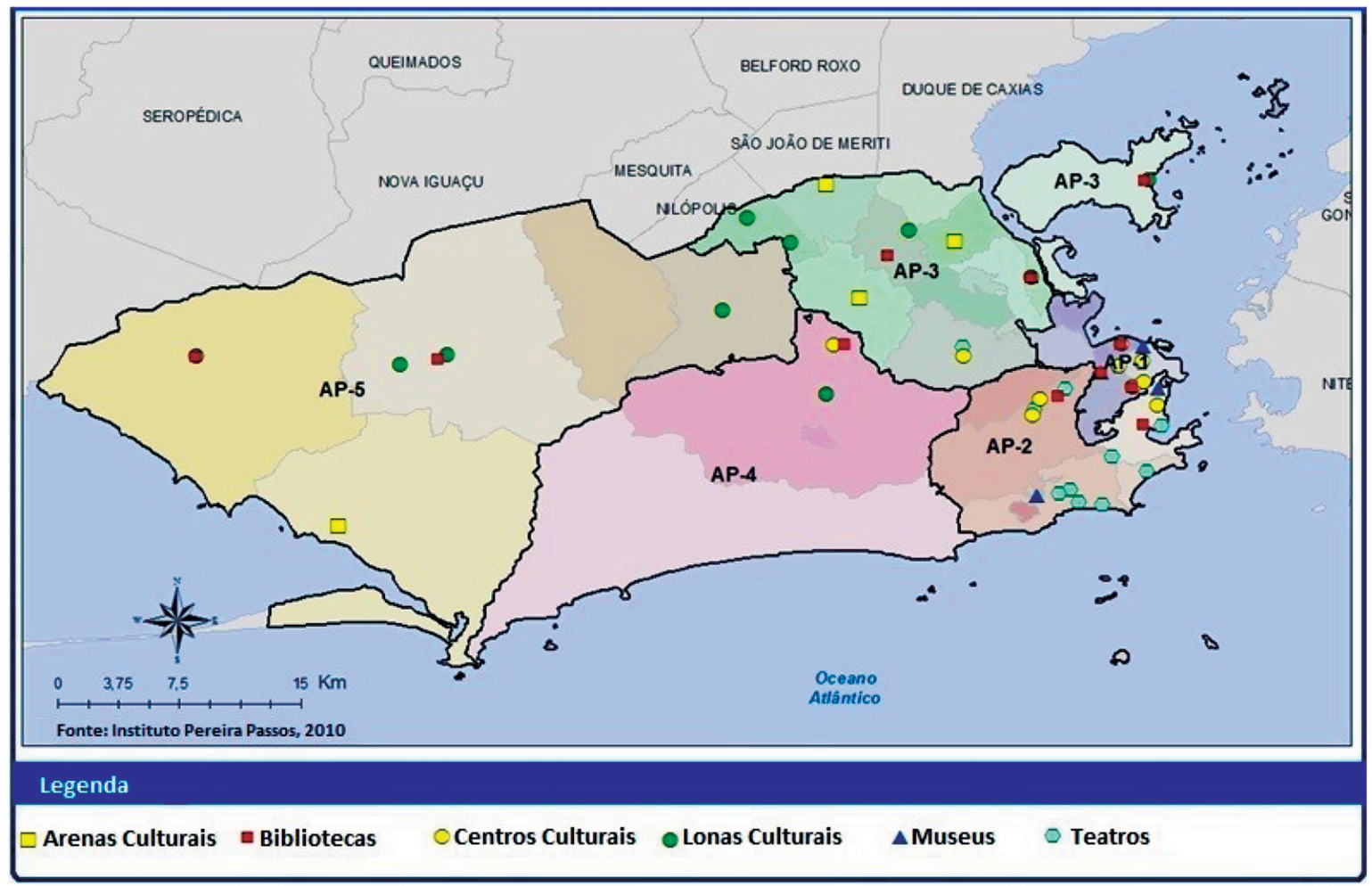

Espaços criados para produção e difusão de diferentes formas de expressão artística, os Centros Culturais são espaços que recebem artes visuais e cênicas, música e cinema, além de palestras e oficinas. Em 2013, eram Centros Culturais da SMC-RJ o Centro Coreográfico da Cidade do Rio de Janeiro, na Tijuca; Centro Municipal de Artes Calouste Gulbenkian, na Praça XI; Centro Cultural Municipal Laurinda Santos Lobo, em Santa Teresa; Centro Cultural Oduvaldo Vianna Filho - Castelinho do Flamengo, no Flamengo; Centro Cultural Parque das Ruínas, em Santa Teresa; Centro Cultural professora Dyla Sylvia de Sá, em Jacarepaguá; Centro de Referência da Música Carioca Artur da Távola, na Tijuca; o Centro Municipal de Artes Hélio Oiticica, no Centro; Centro Cultural Municipal João Nogueira, no Méier; e Centro Cultural José Bonifácio (fechado em função de interdições para as obras na região da Praça Mauá) (SMC, s/d).

As Lonas Culturais são equipamentos estruturados para receber apresentações de artes cênicas e música com acomodações em arquibancadas completadas por cadeiras. Em 2013, faziam parte da estrutura da SMC-RJ as Lonas Culturais Elza Osborne, em Campo Grande; Gilberto Gil, em Realengo; Hermeto Pascoal, em Bangu; Carlos Zéfiro, em Anchieta; João Bosco, em Vista Alegre; Terra, em Guadalupe; Sandra de Sá, em Santa Cruz; Herbert Vianna, na Maré; Jacob do Bandolim, em Jacarepaguá; e Renato Russo, na Ilha do Governador (SMC, 2010a). 
Os Museus sob gestão da Secretaria Municipal de Cultura são equipamentos culturais que ajudam a recontar a história da cidade em diferentes contextos sócio-históricos. Em 2013, eram Museus da Secretaria de Cultura o Ecomuseu de Santa Cruz; Memorial Municipal Getúlio Vargas, na Glória; o Museu Histórico da Cidade do Rio de Janeiro, na Gávea (naquele ano fechado ao público); e o MAR - Museu de Arte do Rio, na Praça Mauá (SMC, 2011a).

Os teatros da SMC-RJ são equipamentos com programação constante, apresentando uma estrutura fundamental para a oferta de espetáculos e experiências cênicas na cidade, com salas de referência e teatros de guignol (fantoches e marionetes). No ano de 2013, a rede de teatros reunia o Espaço Cultural Sérgio Porto, no Humaitá; o Teatro Café Pequeno, no Leblon; os teatros Maria Clara Machado e do Jockey, na Gávea; a sala Baden Powel, em Copacabana; o Teatro Ziembinski, na Tijuca; o Teatro Gonzaguinha, na Praça XI; o Teatro Ipanema, em Ipanema; o Teatro Carlos Gomes, no Centro; os teatros de Guignol do Méier e da Tijuca, e o Teatro de Fantoches e Marionetes Carlos Werneck de Carvalho, no Flamengo (SMC, 2010b; 2011b; DIAS, 2012).

A estrutura disponível para oferta cultural, no ano de 2013, apontava as condições estruturais existentes para a distribuição da programação, através da qual se concretizou a oferta. Deve-se considerar que as políticas de construção da programação dos equipamentos culturais, os mecanismos de diálogo com os públicos, entre outros fatores, são aspectos relevantes nos processos de análise que levem em conta a complexidade do cenário e busquem identificar oportunidades de dinamização dos mecanismos compensatórios para os limites físicos. Contudo, dados sobre estes aspectos, em geral, podem não existir, não estar disponíveis ou ainda não ser observados.

\section{Materiais e Métodos}

O conjunto de dados utilizado no desenvolvimento dos indicadores corresponde aos dados de registros administrativos sobre a capacidade dos equipamentos culturais sob a gestão da Secretaria Municipal de Cultura do Rio de Janeiro (SMC-RJ), as atividades realizadas e o público neles atendido, entre janeiro e dezembro de 2013. São dados cedidos pela SMC-RJ sobre os cinqüenta e dois equipamentos culturais que integravam a estrutura para oferta cultural na cidade naquele ano. Os dados apresentam informações sobre as 17.529 atividades realizadas, distribuídas entre as dez linhas de ação que estruturavam as diretrizes das políticas culturais da cidade naquele período, referentes à teatro, circo, música, dança, artes visuais, incentivo ao hábito da leitura, publicação de estudos, pesquisas, ensaios e obras literárias diversas sobre a cultura e a economia criativa cariocas, realização de espetáculos e intervenções em espaços públicos, realização de mostras, festivais, mercados, feiras e premiações culturais e atividades gerais, que compreendem desde ações sociais a encontros, eventos comemorativos, multiplataforma, entre outros (DANTAS, 2015).

Para a organização, processamento e apresentação dos dados e resultados foi utilizado o Excel, do pacote Office da Microsoft, e o ambiente de geoprocessamento ArcGIS v.10. 0 indicador sintético ${ }^{6}$ foi construído através da aplicação de métodos estatísticos com emprego de técnicas de geoprocessamento para a representação espacial dos resultados.

\footnotetext{
6 Entre os tipos de indicadores, são chamados de índices (ou indicadores sintéticos) aqueles traduzem uma relação entre variáveis, como traduz a proposta do IOC. Assim, conceitualmente, o nome mais apropriado para ele seria Índice de Oferta Cultural. Contudo, no processo de desenvolvimento da pesquisa, foi utilizada continuamente a expressão "indicador sintético" com a supressão da palavra "sintética" para a melhor comunicação. Desta forma, o nome completo do IOC, como resultado do desenvolvimento da pesquisa, deveria ser "Indicador Sintético de Oferta Cultural"; mas visando a simplificação da comunicação, utilizou-se Indicador de Oferta Cultural.
} 
Em função do perfil do conjunto de dados, com um número significativo de variáveis com valor zero e valores extremos, o uso de modelagem estatística seria comprometido. Assim, a proposta metodológica para a construção dos indicadores sobre oferta cultural (por Áreas de Planejamento e Regiões Administrativas) consiste no uso de técnica estatística descritiva com distribuição de proporções de acordo com a quantidade de objetos em cada uma das classes delimitadas, referentes aos equipamentos culturais, a saber: atividade e capacidade. Isso contribuiu para a apresentação dos resultados através dos recortes geográficos por Áreas de Planejamento (APs) e Regiões Administrativas (RAs) da cidade, favorecendo desagregação dos resultados e o detalhamento das análises também em relação aos indicadores complementares. Desta forma, a metodologia traduz a quantificação de aspectos da dinâmica cultural através de indicadores que permitam a visualização da distribuição da fruição cultural, em 2013, nos equipamentos culturais sob a gestão da Secretaria Municipal de Cultura do Rio de Janeiro em escalas diferentes e graus de especificidade. Usando proporções, propôs-se também um sistema complementar de indicadores que ampliou as análises centrais, a partir dos indicadores sobre oferta cultural (DANTAS, 2015).

$\mathrm{Na}$ construção do Indicador de Oferta Cultural (IOC), seguiram-se duas fases: (1) uniformização do conjunto de dados; e (2) ponderação dos indicadores a partir dos pesos atribuídos. Na uniformização, foram considerados os Equipamentos Culturais (i), seus Atributos de Medição ( $j$ ) e a dimensão geográfica ( $k$ ) (DANTAS, 2015), representada por:

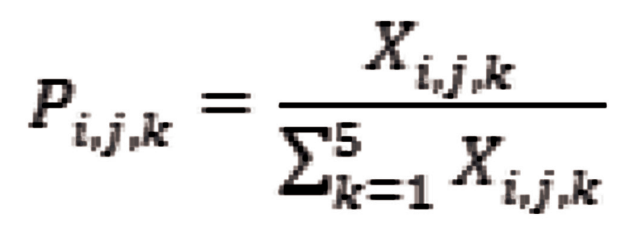

Com isso, todas as observações assumem a mesma escala, variando entre 0 e 1 , para representar os atributos de medição de cada equipamento cultural (EC). A isso, seguiu-se à ponderação, em função do número de Equipamentos Culturais (i) e de seus Atributos de Medição $(j)$, onde $n(i){ }^{*} n(j)$ representa os pesos atribuídos para o cálculo do indicador (DANTAS, 2015), representado por:

$$
\mathrm{IOC}_{\mathrm{i}, \mathrm{j}, \mathrm{k}}=\mathrm{P}_{\mathrm{i}, \mathrm{j}, \mathrm{k}} * \frac{1}{n(i) * n(j)}
$$

Visto que o indicador tem distribuição igual, o fator utilizado na ponderação foi uma constante (W) de valor igual a, aproximadamente, 0,0833, e a representação para o cálculo das parcelas dos atributos do indicador (DANTAS, 2015) é representada por:

$$
\mathrm{IOC}_{\mathrm{i}, \mathrm{j}, \mathrm{k}}=\mathrm{P}_{\mathrm{i}, \mathrm{j}, \mathrm{k}} * W
$$

Concluídos os processos de uniformização e ponderação e compreendendo que j é um atributo que compõe a informação do equipamento cultural, as parcelas são reunidas representando o indicador final por $I O C_{1, \mathrm{k}}=I O C_{\mathrm{i}, 1, \mathrm{k}}+\mathrm{IOC}_{\mathrm{i}, 2, \mathrm{k}}$. Assim, sendo $I O C_{1,1}=I O C_{1,1,1}+$ $I O C_{1,2,1} ; \ldots ; I_{6,5}=I_{6,1,5}+I_{6,2,5}$, o indicador final é representado na Tabela 1 (DANTAS, 2015): 
Tabela 1: Indicador de Oferta Cultural por Área de Planejamento dos Equipamentos sob a gestão da Secretaria Municipal de Cultural do Rio de Janeiro

\begin{tabular}{lccccc}
\hline \multicolumn{1}{c}{$\begin{array}{c}\text { Equipamento } \\
\text { Cultural (i) }\end{array}$} & \multicolumn{5}{c}{ Áreas de Planejamento (k) } \\
\cline { 2 - 6 } 1. Arena Cultural & $\mathbf{1}$ & $\mathbf{2}$ & $\mathbf{3}$ & $\mathbf{4}$ & $\mathbf{5}$ \\
2. Biblioteca & $\mathrm{IOC}_{1,1}$ & $\mathrm{IOC}_{1,2}$ & $\mathrm{IOC}_{1,3}$ & $\mathrm{IOC}_{1,4}$ & $\mathrm{IOC}_{1,5}$ \\
3. Centro Cultural & $\mathrm{IOC}_{2,1}$ & $\mathrm{IOC}_{2,2}$ & $\mathrm{IOC}_{2,3}$ & $\mathrm{IOC}_{2,4}$ & $\mathrm{IOC}_{2,5}$ \\
4. Lona Cultural & $\mathrm{IOC}_{3,1}$ & $\mathrm{IOC}_{3,2}$ & $\mathrm{IOC}_{3,3}$ & $\mathrm{IOC}_{3,4}$ & $\mathrm{IOC}_{3,5}$ \\
5. Museu & $\mathrm{IOC}_{4,1}$ & $\mathrm{IOC}_{4,2}$ & $\mathrm{IOC}_{4,3}$ & $\mathrm{IOC}_{4,4}$ & $\mathrm{IOC}_{4,5}$ \\
6. Teatro & $\mathrm{IOC}_{5,1}$ & $\mathrm{IOC}_{5,2}$ & $\mathrm{IOC}_{5,3}$ & $\mathrm{IOC}_{5,4}$ & $\mathrm{IOC}_{5,5}$ \\
\hline Por AP & $\mathrm{IOC}_{6,1}$ & $\mathrm{IOC}_{6,2}$ & $\mathrm{IOC}_{6,3}$ & $\mathrm{IOC}_{6,4}$ & $\mathrm{IOC}_{6,5}$ \\
\hline
\end{tabular}

Fonte: DANTAS, 2015.

Tendo em vista que o objeto é analisado nas escalas de Áreas de Planejamento e de Regiões Administrativas, a aplicação da formulação do indicador para Regiões Administrativas será análoga à aplicação para Áreas de Planejamento. Utilizando-se os dados referentes à Capacidade e Atividade, para Área de Planejamento e Região Administrativa, tem-se o Indicador de Oferta Cultural para ambas as escalas.

Reconhecendo a importância de uma análise que considere o maior número de possibilidades que o conjunto de dados disponível proporcione, propôs-se a construção de indicadores que possibilitassem a análise de outros aspectos da oferta cultural. Para isso, foram utilizados os dados sobre atividade, capacidade e público referentes às cinco áreas de planejamento (APs), trinta e três regiões administrativas (RAs) e cinquenta e dois equipamentos culturais (ECs). O sistema proposto é composto por vinte e nove indicadores que permitem análises sobre Atividade, Capacidade e Público em relação ao mesmo tipo de equipamento cultural por Área de Planejamento e Região Administrativa; em relação a todos os equipamentos culturais da mesma Área de Planejamento e Região Administrativa e em relação a toda a cidade também por Área de Planejamento e Região Administrativa. Os indicadores do sistema também apresentam resultados sobre público médio por Área de Planejamento e Região Administrativa; e Uso Eficiente por Área de Planejamento e Região Administrativa. Compõe ainda o sistema proposto, indicadores relacionados aos Equipamentos Culturais individualmente, como Indicador de Oferta Cultural, Capacidade, Atividade, Capacidade Disponibilizada no ano, Público, Público Médio e Uso Eficiente (DANTAS, 2015).

A partir dos materiais e métodos utilizados, os resultados alcançados intentam permitir análises dos fenômenos observados comparando-se o resultado do Indicador de Oferta Cultural para as APs (IOC-AP) e RAs (IOC-RA) da cidade, somados aos indicadores do sistema proposto. Relacionando os diferentes indicadores do sistema, as possibilidades de análise oferecem contribuições oportunizadas pelo uso de um conjunto mais robusto de informações nas avaliações realizadas e considerações sobre resultados.

\section{Resultados e Análises}

Os resultados do cálculo do Indicador de Oferta Cultural por Área de Planejamento (IOCAP), apresentados na Tabela 2, confirmam o desequilíbrio na distribuição espacial da oferta cultural na cidade no ano de 2013 . Verifica-se que as APs $1(25,92 \%)$ e $2(24,72 \%)$ concentram proporções elevadas da oferta cultural no período em análise, mas é a AP3 (34,46\%) que apresenta maior proporção da oferta cultural nos equipamentos culturais (ECs) da SMC-RJ naquele ano. 
As Áreas de Planejamento que compõem a zona Oeste da cidade não alcançam $15 \%$ da oferta cultural da cidade no período em análise. A AP5 circunscrevia 10,96\% da oferta cultural, enquanto AP4 registrava 3,94\%. Analisando proporção da oferta cultural das APs 4 e 5 em comparação com proporções relativas aos ECs das outras APs, vê-se que a oferta cultural nestas duas regiões é reduzida em relação ao que se verifica em outras regiões da cidade.

Tabela 2: Indicador de Oferta Cultural por Área de Planejamento (IOC-AP), município do Rio de Janeiro, 2013 (\%)

\begin{tabular}{lccccc}
\hline \multicolumn{1}{c}{$\begin{array}{c}\text { Equipamento } \\
\text { Cultural }\end{array}$} & $\mathbf{1}$ & $\mathbf{2}$ & $\mathbf{3}$ & $\mathbf{4}$ & $\mathbf{5}$ \\
\cline { 2 - 6 } 1. Arena Cultural & 0,00 & 0,00 & 12,89 & 0,00 & 3,77 \\
2. Biblioteca & 2,24 & 4,75 & 6,63 & 1,81 & 1,23 \\
3. Centro Cultural & 6,95 & 4,19 & 4,46 & 1,07 & 0,00 \\
4. Lona Cultural & 0,00 & 0,00 & 9,65 & 1,06 & 5,96 \\
5. Museu & 13,26 & 3,41 & 0,00 & 0,00 & 0,00 \\
6. Teatro & 3,47 & 12,37 & 0,83 & 0,00 & 0,00 \\
\hline Por AP & 25,92 & 24,72 & 34,46 & 3,94 & 10,96 \\
\hline
\end{tabular}

A oferta cultural da AP5 (10,96\%) é menor do que a oferta cultural de alguns tipos de equipamento cultural (EC) de outras Áreas de Planejamento da cidade, como os Museus da AP1 $(13,26 \%)$, os Teatros da AP2 (12,37\%) e das Arenas Culturais da AP3 $(12,89)$. Também em relação a equipamentos culturais de outras APs, a oferta cultural da AP4 só não é menor que as Bibliotecas das APs $1(2,24 \%)$ e $5(1,23 \%)$, do Teatro da AP3 $(0,83 \%)$ e dos Teatros da AP1 (3,47\%), dos Museus da AP2 (3,41\%) e da Arena da AP5 (3,77\%), registrando valor bem próximo dos três últimos. Isso permite dimensionar a proporção da disponibilidade de oferta cultural em algumas regiões da cidade, na estrutura pública municipal, reconhecendo o desequilíbrio inclusive entre algumas regiões e equipamentos culturais de outras regiões da cidade.

O cálculo do Indicador de Oferta Cultural por Região Administrativa (IOC-RA) evidencia que, observando os resultados mais desagregados, confirma-se o desequilíbrio na distribuição espacial da oferta cultural também entre as diferentes Regiões Administrativas (RAs) de cada Área de Planejamento (AP), no ano de 2013. Quando são analisadas as 33 RAs, nota-se que algumas Regiões concentram parte da oferta cultural das diferentes APs, enquanto outras chegam a não apresentar registro de oferta cultural ou apresentam registro em proporções bem inferiores a outras, como se pode verificar nas Tabelas 3, 4 e 5.

É possível verificar, na Tabela 3 (sobre as Regiões Administrativas das Áreas de Planejamento 1 e 2), a inexistência equipamentos culturais nas RAs VII e XXI da AP1 e nas RAs IX e XXVII da AP2. Notam-se ainda RAs com registros de oferta cultural baixos como são a RA I (0,59\%), na AP1, e a RA V (1,98\%), na AP2. Em contraponto, verificam-se RAs em ambas as APs registrando valores mais elevados, como a RA II (17,42\%), na AP1, e as RAs IV (9,88\%) e VIII (7,32\%), na AP2.

Reconhece-se que, assim como se identificou nas análises sobre a distribuição da oferta cultural das APs, algumas RAs registram proporção menor do que alguns tipos de equipamentos culturais de outras RAs. Isso se verifica nos casos da RA I (0,59\%) em relação aos Centros Culturais das RAs II e III (respectivamente 1,07\% e 2,45\%), por exemplo, e da RA V (1,98\%) em relação aos Teatros das RAs IV e VI (respectivamente 2,55\% e 5,54\%), por exemplo. A verificação de desequilíbrios na distribuição da oferta cultural é confirmada entre as RAs em todas as APs com padrões similares aos ilustrados nas análises dos resultados do IOC-AP. 
Tabela 3: Indicador de Oferta Cultural por Região Administrativa (IOC-RA) das Áreas de Planejamento 1 e 2, município do Rio de Janeiro, 2013 (\%)

\begin{tabular}{|c|c|c|c|c|c|c|c|c|c|c|c|c|}
\hline \multirow{3}{*}{$\begin{array}{l}\text { Equipamento } \\
\text { Cultural }\end{array}$} & \multicolumn{12}{|c|}{ Divisões Administrativas } \\
\hline & \multicolumn{6}{|c|}{ AP1 } & \multicolumn{6}{|c|}{ AP2 } \\
\hline & RA.I & RA.II & RA.III & RA.VII & RA.XXI & RA.XXIII & RA.IV & RA.V & RA.VI & RA.VIII & RA.IX & RA.XXVII \\
\hline Arena & 0,00 & 0,00 & 0,00 & 0,00 & 0,00 & 0,00 & 0,00 & 0,00 & 0,00 & 0,00 & 0,00 & 0,00 \\
\hline Biblioteca & 0,59 & 0,00 & 0,73 & 0,00 & 0,00 & 0,92 & 2,75 & 0,00 & 0,00 & 2,01 & 0,00 & 0,00 \\
\hline $\begin{array}{l}\text { Centro } \\
\text { Cultural }\end{array}$ & 0,00 & 1,07 & 2,45 & 0,00 & 0,00 & 3,43 & 1,17 & 0,00 & 0,00 & 3,02 & 0,00 & 0,00 \\
\hline Lona & 0,00 & 0,00 & 0,00 & 0,00 & 0,00 & 0,00 & 0,00 & 0,00 & 0,00 & 0,00 & 0,00 & 0,00 \\
\hline Museu & 0,00 & 12,87 & 0,39 & 0,00 & 0,00 & 0,00 & 3,41 & 0,00 & 0,00 & 0,00 & 0,00 & 0,00 \\
\hline Teatro & 0,00 & 3,47 & 0,00 & 0,00 & 0,00 & 0,00 & 2,55 & 1,98 & 5,54 & 2,29 & 0,00 & 0,00 \\
\hline Total & 0,59 & 17,42 & 3,57 & 0,00 & 0,00 & 4,34 & 9,88 & 1,98 & 5,54 & 7,32 & 0,00 & 0,00 \\
\hline
\end{tabular}

Considerando a distribuição de Equipamentos Culturais nas Regiões Administrativas, seria passível de crença compreender a distribuição não harmônica da oferta cultural no território, visto que os diferentes tipos de equipamentos culturais não estão presentes de forma equânime nas diferentes Regiões Administrativas e Áreas de Planejamento. Contudo, vê-se que equipamentos culturais com a mesma capacidade não apresentaram valores iguais no resultado do IOC (tanto quando observado por AP quanto por RA), sugerindo que fatores que impliquem no volume de atividades realizadas também possam ter contribuições não desprezíveis na orientação do resultado.

Isto é verificável quando se observam, por exemplo, as Arenas Culturais (Tabela 4), que são equipamentos com capacidade igual ou bem parecida, com presença de apenas uma Arena em cada uma das RAs onde este tipo de EC existe. Em casos como estes, a informação expressa através dos resultados pode servir de norte para a construção de perguntas que orientem reflexões aos gestores das unidades, mas também do conjunto de equipamentos culturais, em um processo de busca por melhoria contínua e otimização das atividades realizadas, pensando também no atendimento aos públicos: de artistas, companhias e grupos, produtores e diferentes perfis de espectadores. Isto pode considerar políticas de ocupação dos equipamentos culturais transversais a outras ações, como fomentos direto e indireto e estratégias de comunicação para ocupação da pauta, entre outras possibilidades.

Ainda sobre o desequilíbrio na oferta cultural entre as diferentes RAs da AP3, considerando apenas as RAs com presença de algum tipo de equipamento cultural, nota-se que os resultados apresentam variações não desprezíveis. Contudo, avaliações sobre o que tais variações podem representar exigem a observação da realidade fundada em um conjunto de informações complementares sobre suas condições.

Tabela 4: Indicador de Oferta Cultural por Região Administrativa (IOC-RA) da Área de Planejamento 3, município do Rio de Janeiro, 2013 (\%)

\begin{tabular}{|c|c|c|c|c|c|c|c|c|c|c|c|c|c|}
\hline \multirow{2}{*}{$\begin{array}{c}\text { Equipamento } \\
\text { Cultural }\end{array}$} & \multicolumn{13}{|c|}{ Divisões Administrativas } \\
\hline & RA.X & RA.XI & RA.XII & RA.XIII & RA.XIV & RA.XV & RA.XX & RA.XXII & RA.XXV & RA.XXVIII & RA.XXIX & RA.XXX & RA.XXXI \\
\hline Arena & 0,00 & 4,95 & 0,00 & 0,00 & 0,00 & 5,09 & 0,00 & 0,00 & 2,85 & 0,00 & 0,00 & 0,00 & 0,00 \\
\hline Biblioteca & 0,00 & 0,00 & 0,00 & 0,00 & 2,65 & 0,00 & 3,32 & 0,00 & 0,00 & 0,00 & 0,00 & 0,67 & 0,00 \\
\hline Centro Cultural & 0,00 & 0,00 & 0,00 & 4,46 & 0,00 & 0,00 & 0,00 & 0,00 & 0,00 & 0,00 & 0,00 & 0,00 & 0,00 \\
\hline Lona & 0,00 & 0,00 & 0,00 & 0,00 & 1,17 & 0,00 & 1,65 & 5,81 & 0,00 & 0,00 & 0,00 & 1,01 & 0,00 \\
\hline Museu & 0,00 & 0,00 & 0,00 & 0,00 & 0,00 & 0,00 & 0,00 & 0,00 & 0,00 & 0,00 & 0,00 & 0,00 & 0,00 \\
\hline Teatro & 0,00 & 0,00 & 0,00 & 0,83 & 0,00 & 0,00 & 0,00 & 0,00 & 0,00 & 0,00 & 0,00 & 0,00 & 0,00 \\
\hline Total & 0,00 & 4,95 & 0,00 & 5,29 & 3,82 & 5,09 & 4,97 & 5,81 & 2,85 & 0,00 & 0,00 & 1,68 & 0,00 \\
\hline
\end{tabular}


Nesse e em outros contextos, o uso de informações adicionais sobre a gestão é importante para que se avalie e faça inferências pautadas em fatos e condições sob as quais aquela realidade se estruturou. Buscar compreender especificidades da realidade em avaliação a partir dos indicadores pautados em dados da realidade, mas usando referenciais complementares imprecisos tende a não contribuir para que se alcancem as melhorias necessárias e desejadas.

Ao observar os resultados do IOC-RA das APs 4 e 5 (Tabela 5) verifica-se que em duas RAs da AP4 não existem registros de oferta cultural. Nota-se que a AP5 é a única Área de Planejamento onde todas as RAs têm pelo menos um tipo de equipamento cultural. É possível ver também que os resultados que traduzem o perfil da oferta cultural nos ECs destas regiões apresentam valores entre $0,86 \%$ (em relação à Lona Cultural na RA XVII) e $3,77 \%$ (em relação à Arena Cultural da RA XXVI). Como observado na análise por Áreas de Planejamento, verifica-se a proporção de oferta cultural nestas Regiões abaixo da média de diferentes tipos de equipamento cultural de outras Regiões Administrativas.

Tabela 5: Indicador de Oferta Cultural por Região Administrativa (IOC-RA) das Áreas de Planejamento 4 e 5, município do Rio de Janeiro, 2013 (\%)

\begin{tabular}{l|c|c|c|r|r|rrr}
\hline \multirow{2}{*}{$\begin{array}{c}\text { Equipamento } \\
\text { Cultural }\end{array}$} & \multicolumn{7}{|c}{ Divisões Administrativas } \\
\cline { 2 - 9 } & \multicolumn{7}{|c|}{ AP4 } & \multicolumn{5}{c}{ AP5 } \\
\cline { 2 - 10 } & RA.XVI & RA.XXIV & RA.XXXIV & RA.XVII & RA.XVIII & RA.XIX & RA.XXVI & RA.XXXIII \\
\hline Arena & 0,00 & 0,00 & 0,00 & 0,00 & 0,00 & 0,00 & 3,77 & 0,00 \\
Biblioteca & 1,81 & 0,00 & 0,00 & 0,00 & 1,23 & 0,00 & 0,00 & 0,00 \\
Centro Cultural & 1,07 & 0,00 & 0,00 & 0,00 & 0,00 & 0,00 & 0,00 & 0,00 \\
Lona & 1,06 & 0,00 & 0,00 & 0,86 & 1,32 & 1,62 & 0,00 & 2,16 \\
Museu & 0,00 & 0,00 & 0,00 & 0,00 & 0,00 & 0,00 & 0,00 & 0,00 \\
Teatro & 0,00 & 0,00 & 0,00 & 0,00 & 0,00 & 0,00 & 0,00 & 0,00 \\
\hline Total & 3,94 & 0,00 & 0,00 & 0,86 & 2,55 & 1,62 & 3,77 & 2,16 \\
\hline
\end{tabular}

Entre os outros equipamentos culturais existentes, nota-se que os resultados concentramse entre $1,06 \%$ e $2,16 \%$ da oferta cultural na cidade naquele ano. Isto pode sugerir que, além de os equipamentos culturais nestas regiões não serem muitos, a oferta cultural ali identificada também é rarefeita.

As análises a partir dos resultados do IOC-AP e do IOC-RA podem permitir que os gestores reconheçam padrões de distribuição e alcance de ações que tenham adotado e que não são perceptíveis ao olhar externo à sua gestão. De posse de documentos e de um conjunto maior de informações de seu cotidiano, os resultados do IOC podem ajudar que reconheçam oportunidades de ajuste das ações, visando alcançar resultados previstos em relação aos objetivos e metas elencados.

Nesse sentido, as contribuições de um sistema de indicadores, dada a importância de uma análise que utilize um conjunto maior e mais diverso de informações, podem incluir análises sobre variáveis que traduzam aspectos que orientem o olhar da gestão para particularidades de suas dinâmicas. Isto pode se estruturar a partir das três variáveis consideradas - atividade, capacidade e público - em relação às unidades geográficas Área de Planejamento e Região Administrativa, assim como em relação a cada um dos Equipamentos Culturais.

Os vinte e nove indicadores propostos complementarmente podem ser utilizados como recursos norteadores de avaliações orientadas à ampliação das possíveis respostas para questões do cotidiano, pautando-se no cruzamento de dados sobre aspectos tanto da 
gestão (em contexto macro) quanto dos equipamentos culturais (em contexto específico, micro). O uso desses indicadores, associados aos resultados do IOC-AP e do IOC-RA, pode se apresentar como recurso para a avaliação de aspectos objetivos e tomadas de decisão, além de favorecer a realização de diagnóstico e apoiar o planejamento, o monitoramento e a avaliação das ações.

O cenário da disponibilidade de dados sobre aspectos comuns e de acordo com padrões que permitam seu uso ainda é incipiente na gestão cultural. Para otimizar o uso dos dados e indicadores é desejável ter maior diversidade de dados e metadados, visto que, para analisar aspectos relevantes sobre as complexidades da gestão cultural, alguns dados podem ainda não ser conhecidos ou mesmo percebidos com o valor que têm como recurso para a gestão.

\section{Algumas considerações}

O uso dos dados da Secretaria Municipal de Cultura do Rio de Janeiro foi importante para a construção dos indicadores e a confirmação da percepção da existência de desequilíbrio da oferta cultural entre as regiões da cidade. Considerando as possibilidades de uso dos resultados, o nível de desagregação dos dados potencializa análises mais robustas, uma vez que possibilita avaliações tanto em diferentes escalas geográficas quanto por equipamento cultural.

O método proposto apresenta-se como recurso de uso viável em diferentes contextos e tipos de gestão com custo baixo e chances de ampliação das análises, em função do perfil e tamanho do conjunto de dados disponível. A composição de um conjunto de indicadores customizado às necessidades da gestão pode permitir que gestor cultural extraia informações que apóiem tomadas de decisão mais seguras com a clareza da relevância dos resultados em um contexto e não como resposta definitiva sobre qualquer aspecto analisado. Isto porque um indicador evidencia informações sobre a gestão em um recorte temporal e gerencial específico, verificando-se mudanças que podem confirmar ou refutar hipóteses em diferentes momentos, em função do que os dados registrem.

As análises baseadas em indicadores sintéticos são relevantes em um contexto mais amplo evidenciando aspectos gerais e fundamentando análises iniciais, bem como sinalizando questões que necessitam de informações mais específicas e demandam tomadas de decisão mais complexas. Isso ratifica a importância da composição de um sistema de indicadores que ajude a pautar avaliações mais detalhadas e atentas, justificando e demandando um compromisso com a organização de dados que podem se constituir como diferenciais gerenciais em médio e longo prazos.

Registra-se a necessidade de cautela com avaliações e tomadas de decisão em cenários complexos, como o da gestão cultural, pautadas em apenas um indicador sem que se busquem informações que ratifiquem ou relativizem seus resultados. Tendo em vista que os indicadores podem ser recursos estratégicos para a gestão, como fontes de informação para tomadas de decisão, é importante ter dados com qualidade e utilizar métodos bem estruturados, buscando ter resultados que favoreçam decisões seguras. 


\section{Referências bibliográficas}

CAVALCANTI, M;GOMES, E. Anova riqueza dasorganizações: os capitais doconhecimento. In: Revista TN Petróleo, [S.I.], v. 3, n. 16, p. 1-10, 2000. Disponível em: <http://portal. crie.coppe.ufrj.br/portal/data/documents/storedDocuments/\{93787CAE-E 94C-45C7992B9403F6F40836\}/\{4348A601-CD08-4972-B822-D00D470C0D4C\}/AN ova Riqueza das Organizações.pdf> Acesso em 18 jun. 2014.

CAVALCANTI, M; GOMES, E. Inteligência Empresarial: um novo modelo de gestão para a nova economia. In: Produção, v.10, n. 2, p.53-64, maio, 2001. Disponível em: 〈www. prod.org.br/files/v10 n2/v10n2a05.pdf> Acesso em 18 jun. 2014.

COELHO, T. Dicionário crítico de política cultural. São Paulo: Iluminuras, 2004.

DANTAS, D. C. Indicadores para análise da oferta cultural na Cidade do Rio de Janeiro: um estudo a partir de dados de registros administrativos da Secretaria Municipal de Cultura no ano de 2013. Dissertação (Mestrado em Estudos Populacionais e Pesquisas Sociais) - Escola Nacional de Ciências Estatísticas, Rio de Janeiro, 2015. 211 f.

A utilização de Métodos Quantitativos aplicados a estudos da área temática da Gestão Cultural: uma revisão sistemática. 2017. 44p Monografia (Especialização em Estatística Aplicada). Instituto de Ciências Exatas, Departamento de Matemática, Universidade Federal Rural do Rio de Janeiro, Seropédica, RJ, 2017. Mimeo.

DIAS, J. da S. Teatros do Rio: do Século XVIII ao Século XX. Rio de Janeiro: FUNARTE, 2012.

FANCHETTE, S. Les indicateurs culturales: theorie et pratique. Reunion d'experts sur lês estatistiques et lês indicateurs culturels. Paris: UNESCO, 1979. Disponível em 〈http://unesdoc.unesco.org/images/0003/000380/038077fb.pdf〉 Acesso em 13 abr. 2013.

JANNUZZI, P. M. Indicadores sociais no Brasil: conceitos, fontes de dados e aplicações. 3. ed. Campinas: Editora Alínea, 2004(a).

Indicadores para diagnóstico, monitoramento e avaliação de

programas sociais no Brasil. Revista do Serviço Público, Brasília, v.58, n.2, p. 137-160, abr/jun, 2005.

MÉDICI, A. C. Sistema estatístico, planejamento e sociedade no Brasil (notas para uma discussão). In: Revista Brasileira de Estudos Populacionais, Campinas, v. 7, n. 2, p. 191-206, 1990. Disponível em <http://www.abep.nepo.unicamp.br/docs/rev_inf/vol7_

n2_1990/vol7_n2_1990_5artigo_191_206.pdf> Acesso em 26 jun. 2013.

MORAIS, M. da P.; LIMA, R. Indicadores urbanos como instrumentos de gestão e formulação de políticas públicas. In.: Revista de Administração Municipal - IBAM. v. 46, n. 232. Nov/Dez, 2001. p.21-27.

PFENNINGER, M. Indicadores y Estadísticas Culturales: Breve repaso conceptual. In: Boletín GC, n. 7, 2004. Disponível em: <http://www.gestioncultural.org/ficheros/1_ 1316771694 _MPfenniger.pdf> Acesso em 13 abr. 2013.

PIOVANI, J. I. Reflexiones metodológicas sobre la evaluación académica. In.: Revista Política Universitaria. Buenos Aires, año 2, N.2, p. 2-11, agosto de 2015. Disponível em 〈http://iec.conadu.org.ar/files/publicaciones/1441904118_2-digital.pdf〉, acesso em 05 setembro 2017.

SECRETARIA MUNICIPAL DE CULTURA-SMC. Arenas. 2013. Disponível em <http://www. rio.rj.gov.br/web/smc/arenas> Acesso em 14 jun. 2015. 
SECRETARIA MUNICIPAL DE CULTURA-SMC. Bibliotecas Populares. 2009. Disponível em 〈http://www.rio.rj.gov.br/web/smc/bibliotecas〉 Acesso em 14 jun. 2015.

SECRETARIA MUNICIPAL DE CULTURA-SMC. Centros Culturais. s/d. Disponível em 〈http://www.rio.rj.gov.br/web/smc/centros-culturais〉 Acesso em 14 jun. 2015.

SECRETARIA MUNICIPAL DE CULTURA-SMC. Lonas Culturais. 2010(a). Disponível em 〈http://wWw.rio.rj.gov.br/web/smc/lonas-culturais〉 Acesso em 14 jun. 2015.

SECRETARIA MUNICIPAL DE CULTURA-SMC. Museus. 2011(a). Disponível em <http:// www.rio.rj.gov.br/web/smc/museus> Acesso em 14 jun. 2015.

SECRETARIA MUNICIPAL DE CULTURA-SMC. Teatros. 2010(b). Disponível em <http:// Www.rio.rj.gov.br/web/smc/teatros> Acesso em 14 jun. 2015.

SECRETARIA MUNICIPAL DE CULTURA-SMC. Teatros de Guignol. 2011(b). Disponível em 〈http://www.rio.rj.gov.br/web/smc/teatros-de-guignol〉 Acesso em 14 jun. 2015.

UNESCO. Declaração Universal sobre a Diversidade Cultural. Paris: UNESCO, 2002. Disponível em: 〈http://unesdoc.unesco.org/images/0012/001271/127160por.pdf〉 Acesso em 12 jan. 2014.

YUE, A; KHAN, R; BROOK, S. Developing a local cultural indicator framework in Australia: a case of the city of Whittlesea. In: Culture and Local Governance, v. 3, n. 1- 2, 2011. Disponível em <https://uottawa.scholarsportal.info/ojs/index.php/clg-cgl/ article /view/191/174> Acesso 16 abr. 2013.

WALLGREN, A., WALLGREN, B. Estadísticas basadas en registros: aprovechamiento estadístico de datos administrativos. México: INEGI, 2012. 